\title{
Dropout-based Active Learning for Regression
}

\author{
Evgenii Tsymbalov, Maxim Panov, and Alexander Shapeev \\ Skolkovo Institute of Science and Technology (Skoltech), \\ Nobel str. 3, Moscow, Russia, 121205, \\ e.tsymbalov@skoltech.ru, m.panov@skoltech.ru, a.shapeev@skoltech.ru
}

\begin{abstract}
Active learning is relevant and challenging for high-dimensional regression models when the annotation of the samples is expensive. Yet most of the existing sampling methods cannot be applied to large-scale problems, consuming too much time for data processing. In this paper, we propose a fast active learning algorithm for regression, tailored for neural network models. It is based on uncertainty estimation from stochastic dropout output of the network. Experiments on both synthetic and realworld datasets show comparable or better performance (depending on the accuracy metric) as compared to the baselines. This approach can be generalized to other deep learning architectures. It can be used to systematically improve a machine-learning model as it offers a computationally efficient way of sampling additional data.
\end{abstract}

Keywords: regression, active learning, uncertainty quantification, neural networks, dropout

\section{Introduction}

Active learning is crucial for the applications in which annotation of new data is expensive. Selection of samples is usually based on uncertainty estimation, by adding the samples on which the prediction is most uncertain to the training set. The standard techniques include the models that estimate their uncertainty directly, such as Bayesian methods [1, or algorithms that estimate uncertainty indirectly, through the deviation of the outputs of an ensemble of models. Models of the first type become computationally expensive when it comes to a large number of samples $\left(>10^{4}\right)$ and input dimensions $(>10)$. Ensemble-like models require full or partial independent training of several models, which is also timeconsuming, even though it can be done in parallel.

At the same time, neural networks can easily handle large amounts of data and thus are widely used in different areas of applied machine learning such as computer vision [2], speech recognition [3], as well as physics [4, manufacturing [5], or chemistry [6]. The popularity of deep learning has increased after the regularization techniques like dropout [7] and optimization procedures [89] have been developed. However, there is still a lack of theoretical understanding about capabilities of neural networks, in particular, estimating model uncertainty.

In our work, we propose a fast active learning algorithm for neural networks for regression problems. Our approach is based on a stochastic output of the 
neural network model, which is performed using different dropout masks at the prediction stage and used to rank unlabeled samples and to choose among them the ones with the highest uncertainty. We demonstrate that our approach has a comparable or better performance with respect to the baselines in a series of numerical experiments.

The rest of the paper is organized as follows. We discuss the related work in Section 2 In Section 3 our MCDUE (Monte-Carlo Dropout Uncertainty Estimation)-based active learning method is described in detail. Section 4 contains numerical experiments on both real-world and synthetic data. Section 5 draws the conclusions to the paper.

\section{Related work}

\subsection{Active learning}

Active learning 10 is a framework in which a machine-learning algorithm may choose the most informative unlabeled data samples, and ask an external oracle to annotate them. In statistics and engineering applications, such a setup is usually referred to as an adaptive design of experiments 1112. In this setup, one has a set (finite or infinite) unlabeled examples, the so-called pool. The function ranking the data points is referred to as an acquisition function or a querying function. Ideally, it should be designed in such a way that adding a relatively small number of data samples helps to improve the performance of the model.

There are numerous approaches to constructing an acquisition function. In the case of Bayesian models, such as the Gaussian Processes 11, model uncertainty may be defined explicitly: various estimates can be adopted to rank data points or even to choose them optimally over the defined region [13[14. However, the major drawback of such models is that calculations are usually intractable for a large number of input dimensions. To cope with this problem various techniques and analogues were proposed, such as Bayesian Neural Networks [15], but all of the methods still remain expensive to train when it comes to a considerably large train set or a complex model.

Another approach is a committee-based active learning [16, also known as query-by-committee, where an ensemble of models is trained on the same or different parts of the data. In this approach the measured inconsistency of the predictions obtained from different models for a given data sample may be considered as an overall ensemble uncertainty. Various investigations in this field include diversification of models [17] and boosting/bagging exploitation [18. Unfortunately, in the case of neural network ensembles (see [19 for a detailed review) this approach often leads to an independent training of several models, which may be computationally expensive for the large-scale applications.

\subsection{Dropout}

Dropout [7]20] is one of the most popular techniques used for a neural network regularization. To put it in plain words, it randomly mutes some of the neu- 
rons in hidden layers during the training stage, forcing them to output zero regardless of an input. This feature of the technique is taken into account during the backpropagation stage of training. Stochastic by nature and simple in implementation, it allows to efficiently reduce overfitting and thus has paved the way for new state-of-the-art results in almost every deep learning application. First proposed as an engineering, empirical approach to reducing the correlation between weights, later it has obtained its theoretical interpretation as an averaged ensembling technique [20, a Bernoulli realization of the corresponding Bayesian neural network 21] and a latent variable model [22]. It was shown in 23] that using dropout at the prediction stage (i.e., stochastic forward passes of the test samples through the network, also referred to as $M C$ dropout) leads to unbiased Monte-Carlo estimates of the mean and the variance for the corresponding Bayesian neural network trained using variational inference. In [23], Gal also proposes and analyses direct estimates for a model uncertainty. We use a lightweight version of this approach, estimating the model uncertainty based on a sample standard deviation (which is, up to a factor, an unbiased model variance estimate) of the stochastic output of the network.

Although there are some applications of the abovementioned approach to active learning setting for classification problems [24 25], to the best of our knowledge, there has been no study of applicability of this approach to the regression task. In this work, we evaluate our MC dropout-based approach and compare it with high-throughput baselines. Our experiments have shown comparable or better accuracy and supremacy in a speed-accuracy trade-off of the proposed approach.

\section{Methodology}

\subsection{Problem statement}

Let

$$
y=f(x), x \in \mathcal{X} \subset \mathbb{R}^{n}, y \in \mathbb{R}
$$

be some unknown function which is to be approximated using the values from the training set

$$
D_{\text {train }}=\left\{x_{j}^{\text {train }}, f\left(x_{j}^{\text {train }}\right), j=1, \ldots, N_{\text {train }}\right\} .
$$

Suppose we have a model (more specifically, a neural network) $\hat{f}: \mathcal{X} \rightarrow \mathbb{R}$ trained on $D_{\text {train }}$ with a mean squared error

$$
L\left(\hat{f}, D_{\text {train }}\right)=\sum_{j=1}^{N_{\text {train }}}\left(f\left(x_{j}^{\text {train }}\right)-\hat{f}\left(x_{j}^{\text {train }}\right)\right)^{2}
$$

as a fitting criterion.

Let us now focus on the setting in which we want to decrease the value of loss function on some set of test points $D_{\text {test }}$ by extending the training set $D_{\text {train }}$ 
with some set of additional samples and performing an additional training of the model $\hat{f}$. More precisely, we are given another set of points called the "pool"

$$
\mathcal{P}=\left\{x_{j}, j=1, \ldots, N_{\text {pool }}, \mathcal{P} \subset \mathcal{X}\right\},
$$

which represents unlabeled data. Each point $x^{*} \in \mathcal{P}$ may be annotated by computing $f\left(x^{*}\right)$ so that the pair $\left\{x^{*}, f\left(x^{*}\right)\right\}$ is added to the training set. We suppose that in a practical application the process of annotation is expensive (i.e., it requires additional resources such as computational time or money), hence we need to choose as few additional points from the set $\mathcal{P}$ as possible to achieve the desired quality of the model. To achieve this goal, we use an acquisition function

$$
A\left(\hat{f}, \mathcal{P}, D_{\text {train }}\right): \mathcal{P} \rightarrow \mathbb{R}_{+},
$$

which ranks the points from $\mathcal{P}$ in such a way that the points with the larger values of $A$ become more appealing for the model to learn on. In the experimental setting of this work, we use a fixed number $m$ of the points to add at each stage of the active learning process.

In most practical applications an acquisition function is related to a model uncertainty, which may be defined in various ways depending on the model and the field. There are also approaches, such as random sampling, that do not use the information from the model $\hat{f}$ (see Section 3.3 for further details). As for computationally heavy models with hundreds of thousands of parameters, such as neural networks, these approaches may be considered as more preferable. In the next section, we introduce a Monte-Carlo Dropout Uncertainty Estimation (MCDUE) approach, which enables us to collect uncertainty information from the neural network.

\subsection{Monte-Carlo Dropout Uncertainty Estimation}

Using dropout at the prediction stage allows us to generate stochastic predictions and, consequently, to estimate the variance of these predictions. Our approach rests on the hypothesis that data samples with higher standard deviations have larger errors of true function predictions. Although this is not always the case (see Figure 1), concerning a neural network of a reasonable size trained on a reasonable number of samples we have observed a clear correlation between dropout-based variance estimates and prediction errors (see Figure2). It should be noted that the result does vary (like any other result of neural network training) depending on several factors: architecture and size of the neural network, samples used for initial training and training hyperparameters, such as regularization, learning rate, and dropout probability. The MCDUE-based active learning algorithm we propose is summarized below.

1. Initialization. Choose a trained neural network $\hat{f}(x)=\hat{f}(x, \omega)$, where $\omega$ is a vector of weights. Set the dropout probability $\pi$. Set the number of stochastic runs $T$.

2. Variance estimation. For each sample $x_{j}$ from the pool $\mathcal{P}$ : 
(a) Make $T$ stochastic runs using dropout of the model $\hat{f}$ and collect outputs $y_{k}=\hat{f}_{k}\left(x_{j}\right)=\hat{f}\left(x_{j}, \omega_{k}\right), k=1, \ldots, T$, where $\omega_{k}$ are sampled from Bernoulli distribution with parameter $\pi$.

(b) Calculate the standard deviation (as an acquisition function):

$$
s_{j}=A^{\operatorname{MCDUE}}\left(x_{j}\right)=\sqrt{\frac{1}{T-1} \sum_{k=1}^{T}\left(y_{k}-\bar{y}\right)^{2}}, \bar{y}=\frac{1}{T} \sum_{k=1}^{T} y_{k} .
$$

3. Sampling. Pick $m$ samples with the largest standard deviations $s_{j}$.

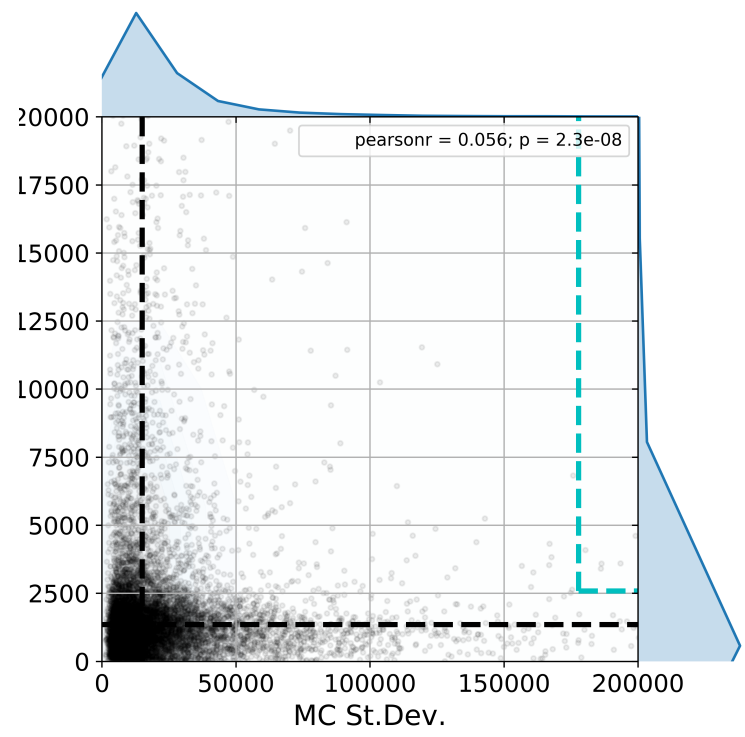

Fig. 1. Scatter plot shows the relation between the MC standard deviation and the absolute error for test samples. The black dashed lines correspond to the medians of distributions, the vertical blue line corresponds to the 0.99 percentile of the MC standard deviation distribution, while the horizontal blue line shows the median percentile of the absolute error distribution of corresponding samples, which is equal to 0.783 in this case. Five-layer neural network with a 256-128-64 structure was used on the Online News Popularity dataset [26]. The Pearson correlation coefficient equals to 0.056, thus showing no linear relation between the absolute error and the MC standard deviation.

In our experiments we used the dropout probability $\pi=0.5$, and number of stochastic runs for each sample $T=25$. We found out that, generally, the decrease in $\pi$ results in an approximately linear scaling of standard deviations $s_{j}$ for not too small $\pi$. The computational cost per one sample is $O\left(T N_{\text {pool }}\right)$. However, on modern GPU-based implementations the sampling can be done 


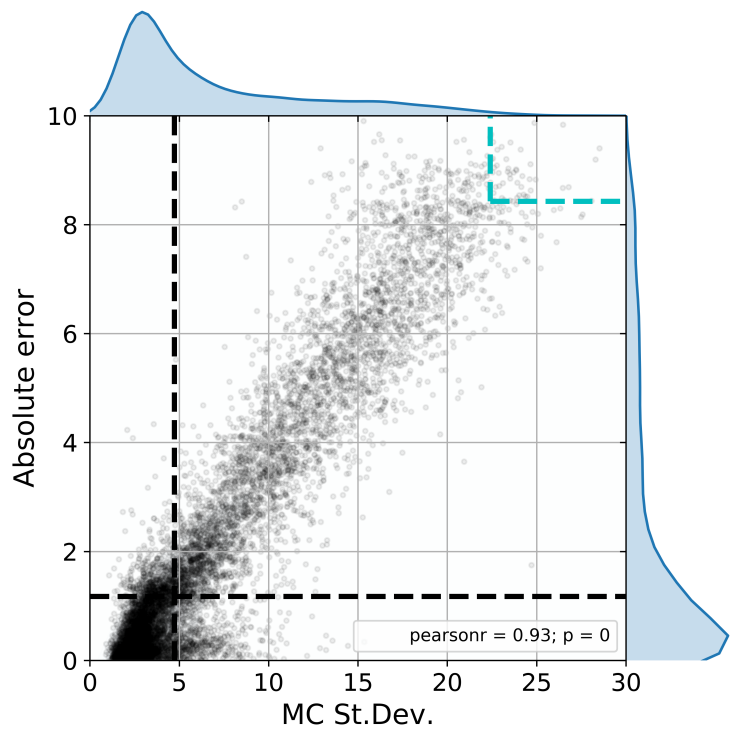

Fig. 2. Scatter plot shows the relation between the MC standard deviation and the absolute error for test samples. The black dashed lines correspond to the medians of distributions, the vertical blue line corresponds to the 0.99 percentile of the MC standard deviation distribution, while the horizontal blue line shows the median percentile of the absolute error distribution of corresponding samples, which is equal to 0.975 in this case. Five-layer neural network with a 256-128-64 structure was used on the CT slices dataset [27. The Pearson correlation coefficient equals to 0.93, thus showing an almost linear relation between the absolute error and the MC standard deviation, so if we choose a sample with a relatively high MC standard deviation, it will probably have large absolute error.

in parallel. One could also decrease $T$ to speed up the procedure. It should be emphasized that we can start with a pre-trained neural network from the previous iteration if we train the model on the extended training set; such a method may significantly speed-up retraining.

\subsection{Baselines}

We use the following baselines to compare the performance.

Random sampling. This algorithm samples random points $x$ from the pool. Computational cost is $O(1)$ in this case, which makes this algorithm the fastest compared to all the others.

Greedy max-min sampling. To sample a point, this algorithm takes the point from the pool most distant from the training set (in the $l_{2}$ sense) and adds it to the training set. This process continues until the required number of points is 
added to the training set. The acquisition function for max-min sampling is

$$
A_{\mathrm{MM}}(x)=\min _{k}\left\|x-x_{k}^{\mathrm{train}}\right\|^{2} .
$$

In this case, computational cost can be estimated as $O\left(N_{\text {train }} N_{\text {pool }}\right)$.

Batch max-min sampling. Although straightforward and intuitive, the maxmin sampling is also computationally expensive in the case of a large number of dimensions and pool/training set size, since it requires that a full distance matrix is calculated on every stage of active learning. We propose the batch version that has the same acquisition function $A_{\mathrm{MM}}$ but samples $K$ points which are the most distant from the training set. Although it does seem less optimal, this solution speeds up sampling up to $K$ times assuming $K$ is the number of samples to be sampled on each iteration. In our experiments, we set $K$ equal to 4 .

\section{Results}

\subsection{Experimental setup}

We focus on the non-Bayesian methods as comparable in terms of the computational time for large datasets and models and compare the active learning algorithms in the following experimental setup:

1. Initialization of the initial dataset $I$, training pool $\mathcal{P}$, number of samples added on each step $m$, the final size of the dataset $f$, network architecture, and learning parameters.

2. The network is trained on the initial dataset $I$. Its weights are copied to the networks corresponding to each active learning algorithm.

3. For each active learning algorithm:

(a) While $|I|<f$ :

i. Obtain the rank $r_{j}$ for every $x_{j} \in \mathcal{P}$ using an acquisition function $A$.

ii. Sample point set $S \subset \mathcal{P},|S|=m$ with maximal ranks $r_{j}$.

iii. Add $S$ to $I: I:=I \bigcup S$.

iv. Exclude $S$ from the corresponding $\mathcal{P}: \mathcal{P}:=\mathcal{P} / S$.

v. Train the neural network on $I$.

(b) Calculate the metrics.

For each experiment, the number of training epochs was set to 10000 . We used the $l_{2}$-regularization of the weights with the regularization parameter $\alpha=10^{-5}$, a five-layer fully-connected network with the 256-128-64 architecture and leaky linear rectifier [28] with leakiness $\beta=0.01$ as an activation function. We used the Theano library [29] and Lasagne framework [30]. Data points were shuffled and split in the following ratio: $20 \%$ on a training set, $60 \%$ on a pool, $20 \%$ on the test set. 


\subsection{Metrics}

During the neural network training, we optimize the mean squared error (MSE, $\left.l_{2}\right)$ metric. However, we also report the mean absolute error (MAE, $l_{1}$ ) and maximum absolute error (MaxAE, $l_{\infty}$ ).

We believe that the actual task of regression is more general than optimization of one given metric (e.g., MSE); thus, the choice of a particular metric is merely an operationalization of the real problem behind the regression task. Moreover, several applications exist, in which the maximal error is a much more appropriate accuracy metric (like chemistry or physical simulations) than the mean error. Unfortunately, it is hard to use the $l_{\infty}$ loss function for training neural network since it is non-differentiable. In case one deals with two algorithms that have a similar MSE and significantly different MaxAE, the algorithm with a smaller maximal error should be preferred.

\subsection{Datasets}

We took the data from UCI ML repository [31, see the Table 1 for more details. All the datasets represent real-world problems with $15+$ dimensions and $30000+$ samples. The exception is the synthetic Rosenbrock 2000D dataset, which has 10000 samples and 2000 dimensions.

Table 1. Summary of the datasets used in our experiments.

\begin{tabular}{|c|c|c|c|}
\hline Dataset name & \# of samples & \# of attributes & Feature to predict \\
\hline BlogFeedback [32] & 60021 & 281 & Number of comments \\
\hline SGEMM GPU [33] & 241600 & 18 & Median calculation time \\
\hline YearPredictionMSD [34 & 515345 & 90 & Year \\
\hline Relative location of CT slices [27] & 53500 & 386 & Relative location \\
\hline Online News Popularity [26] & 39797 & 61 & Number of shares \\
\hline KEGG Network 35] & 53414 & 24 & Clustering coefficient \\
\hline Rosenbrock 2000D [36] & 10000 & 2000 & Function value \\
\hline
\end{tabular}

Since the neural network training procedure is stochastic by its nature, we conducted 20 experiments shuffling the dataset and re-initializing the weights each time.

\subsection{Ratio plots}

First, we compare our MCDUE-based approach with the baseline approaches using the ratio of errors in various metrics. Figures 3 and 4 show that our active learning approach has a better performance than random sampling in RMSE and MaxAE metrics, and a small accuracy increase as compared to a max-min algorithm. It should be noted that as the number of active learning iterations (new data gathering and learning on the top of it) increases (thus leaving the data pool empty), ratio turns to 1. 

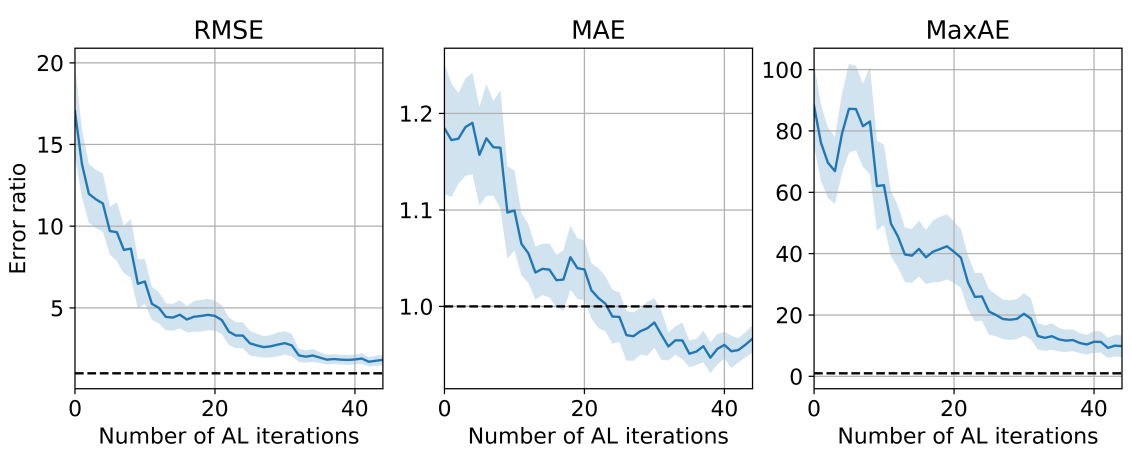

Fig. 3. Comparison of MCDUE-based algorithm and random sampling algorithm: the ratio of errors (training curves) for various metrics on the KEGG Network dataset [35]. Ratio bigger than 1 (dashed line) shows the superiority of the MCDUE-based algorithm. The blue line shows the mean over 25 experiments, the standard deviation is also shown. One can see that the proposed algorithm outperforms the random sampling significantly on RMSE and MaxAE metrics.
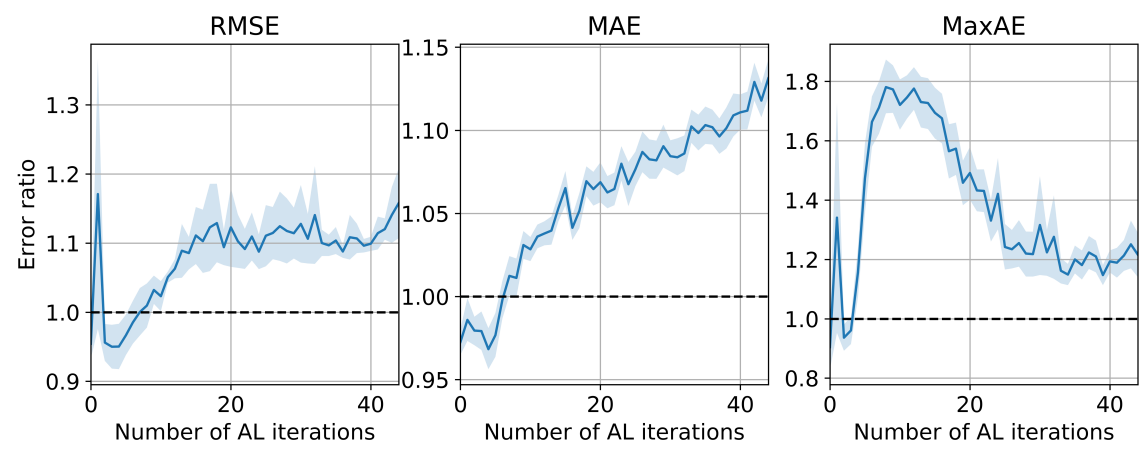

Fig. 4. Comparison of MCDUE-based algorithm and max-min sampling algorithm: the ratio of errors (training curves) for various metrics on KEGG Network dataset 35. Ratio bigger than 1 (dashed line) shows the superiority of the MCDUE-based algorithm. The blue line shows the mean over 25 experiments, the standard deviation is also shown. One can see that the proposed algorithm slightly outperforms the maxmin sampling across all the metrics by up to $20 \%$.

\subsection{Dolan-More plots}

To compare the performance of the algorithms across the different datasets (see Table 1), the initial number of training samples and training samples themselves, we will use Dolan-More curves, which, following [37, may be defined as follows. Let $q_{a}^{p}$ be an error measure of the $a$-th algorithm on the $\mathcal{P}$-th problem. Then, defining the performance ratio $r_{a}^{p}=\frac{q_{a}^{p}}{\min _{x}\left(q_{x}^{p}\right)}$, we can define the Dolan-More 
curve as a function of the performance ratio factor $\tau$ :

$$
\rho_{a}(\tau)=\frac{\#\left(p: r_{a}^{p} \leq \tau\right)}{n_{p}},
$$

where $n_{p}$ is a total number of evaluations for the problem $p$. Thus, $\rho_{a}(\tau)$ defines the fraction of problems in which the $a$-th algorithm has the error not more than $\tau$ times bigger than the best competitor in the chosen performance metric.

We conducted a number of experiments with one iteration of active learning performed on the datasets from Table 1, see Figure 5 for Dolan-More curves. Note that $\rho_{a}(1)$ is the ratio of problems on which the $a$-th algorithm performance was the best, and it is always the case of the MCDUE-based algorithm. Judging by the area under curve (AUC) metric, the MCDUE-based approach outperforms the random sampling and is slightly better than a batch max-min sampling.
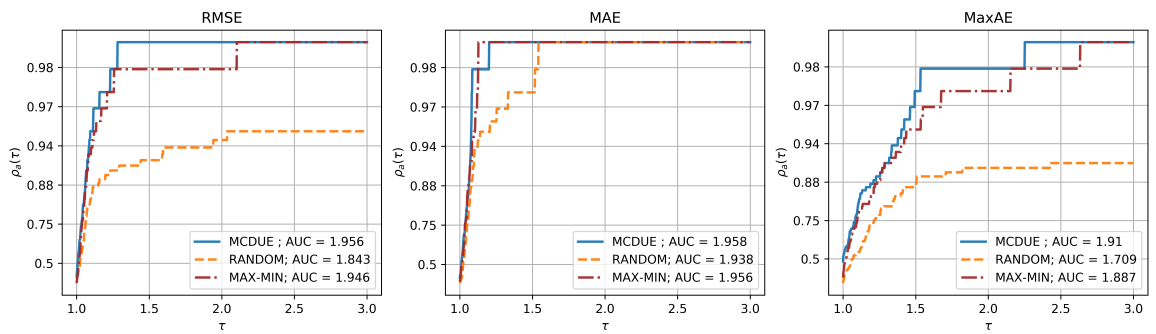

Fig. 5. Log-scaled Dolan-More curves for various metrics and acquisition functions. Figures on the legend indicate the area under the curve (AUC) metric for each algorithm. The number of training samples was chosen randomly from 1000 to the $20 \%$ of the training set, the number $m$ of points to sample from the pool $\mathcal{P}$ was chosen randomly from 100 to 1100 , with a 140 experiments conducted in total. The MCDUEbased approach outperforms the random sampling and is slightly better than batch max-min sampling.

\section{Summary and discussion}

We have proposed an MCDUE-based (Monte-Carlo Dropout Uncertainty Estimationbased) approach to active learning for the regression problems. This approach allows neural network models to estimate self-uncertainty of unlabeled data samples. Numerical experiments on real-life datasets have shown that our algorithm outperforms both random sampling and the max-min baseline. Compared to the latter, our algorithm is faster and relies on the information provided by the model.

Theoretical connections with Bayesian neural networks require further investigations. In this empirical study we propose an ad hoc, lightweight approach 
that may be adopted to strengthen the existing models. In future work, several factors that may affect the results, among which are network architecture, regularization, and dropout probability, require further in-depth study. Another significant improvement would be in a model could estimate the number of samples to acquire on each step.

\section{Acknowledgements}

The work was supported by the Skoltech NGP Program No. 2016-7/NGP (a Skoltech-MIT joint project).

\section{References}

1. Rasmussen, Carl Edward. "Gaussian processes in machine learning." Advanced lectures on machine learning. Springer, Berlin, Heidelberg, 2004. 63-71.

2. Szegedy, Christian, et al. "Inception-v4, inception-resnet and the impact of residual connections on learning." AAAI. Vol. 4. 2017.

3. Sainath, Tara N., et al. "Deep convolutional neural networks for LVCSR." Acoustics, speech and signal processing (ICASSP), 2013 IEEE international conference on. IEEE, 2013.

4. Baldi, Pierre, Peter Sadowski, and Daniel Whiteson. "Searching for exotic particles in high-energy physics with deep learning." Nature communications 5 (2014): 4308.

5. Anjos, Oflia, et al. "Neural networks applied to discriminate botanical origin of honeys." Food chemistry 175 (2015): 128-136.

6. Schtt, Kristof T., et al. "Quantum-chemical insights from deep tensor neural networks." Nature communications 8 (2017): 13890.

7. Hinton, Geoffrey E., et al. "Improving neural networks by preventing co-adaptation of feature detectors." arXiv preprint arXiv:1207.0580 (2012).

8. Tieleman, Tijmen, and Geoffrey Hinton. "Lecture 6.5-rmsprop: Divide the gradient by a running average of its recent magnitude." COURSERA: Neural networks for machine learning 4.2 (2012): 26-31.

9. Kingma, Diederik P., and Jimmy Ba. "Adam: A method for stochastic optimization." arXiv preprint arXiv:1412.6980 (2014).

10. Settles, Burr. "Active learning." Synthesis Lectures on Artificial Intelligence and Machine Learning 6.1 (2012): 1-114.

11. Fedorov, Valerii. Theory of optimal experiments. Elsevier, 1972.

12. Forrester, Alexander, and Andy Keane. Engineering design via surrogate modelling: a practical guide. John Wiley \& Sons, 2008.

13. Sacks, Jerome, et al. "Design and analysis of computer experiments." Statistical science (1989): 409-423.

14. Burnaev, Evgeny, and Maxim Panov. "Adaptive design of experiments based on gaussian processes." International Symposium on Statistical Learning and Data Sciences. Springer, Cham, 2015.

15. Neal, Radford M. Bayesian learning for neural networks. Vol. 118. Springer Science \& Business Media, 2012.

16. Seung, H. Sebastian, Manfred Opper, and Haim Sompolinsky. "Query by committee." Proceedings of the fifth annual workshop on Computational learning theory. ACM, 1992. 
17. Melville, Prem, and Raymond J. Mooney. "Diverse ensembles for active learning." Proceedings of the twenty-first international conference on Machine learning. ACM, 2004.

18. Mamitsuka, Naoki Abe Hiroshi. "Query learning strategies using boosting and bagging." Machine learning: proceedings of the fifteenth international conference (ICML98). Vol. 1. Morgan Kaufmann Pub, 1998.

19. Li, Hui, Xuesong Wang, and Shifei Ding. "Research and development of neural network ensembles: a survey." Artificial Intelligence Review 49.4 (2018): 455-479.

20. Srivastava, Nitish, et al. "Dropout: A simple way to prevent neural networks from overfitting." The Journal of Machine Learning Research 15.1 (2014): 1929-1958.

21. Gal, Yarin, and Zoubin Ghahramani. "Dropout as a Bayesian approximation: Representing model uncertainty in deep learning." international conference on machine learning. 2016.

22. Maeda, Shinichi. "A bayesian encourages dropout." arXiv preprint arXiv:1412.7003 (2014).

23. Gal, Yarin. "Uncertainty in deep learning." University of Cambridge (2016).

24. Kampffmeyer, Michael, Arnt-Brre Salberg, and Robert Jenssen. "Semantic segmentation of small objects and modeling of uncertainty in urban remote sensing images using deep convolutional neural networks." CVPRW IEEE Conference, 2016.

25. Gal, Yarin, Riashat Islam, and Zoubin Ghahramani. "Deep bayesian active learning with image data." arXiv preprint arXiv:1703.02910 (2017).

26. Fernandes, Kelwin, Pedro Vinagre, and Paulo Cortez. "A proactive intelligent decision support system for predicting the popularity of online news." Portuguese Conference on Artificial Intelligence. Springer, Cham, 2015.

27. Graf, Franz, et al. "2D image registration in CT images using radial image descriptors." International Conference on Medical Image Computing and ComputerAssisted Intervention. Springer, Berlin, Heidelberg, 2011.

28. Maas, Andrew L., Awni Y. Hannun, and Andrew Y. Ng. "Rectifier nonlinearities improve neural network acoustic models." Proc. icml. Vol. 30. No. 1. 2013.

29. Al-Rfou, Rami, et al. "Theano: A Python framework for fast computation of mathematical expressions." arXiv preprint arXiv:1605.02688 472 (2016): 473.

30. Dieleman, Sander, et al. "Lasagne: First release., August 2015." URL http://dx doi. org/10.5281/zenodo 27878 (2016).

31. Dua, D. and Karra Taniskidou, E. (2017). UCI Machine Learning Repository http://archive.ics.uci.edu/ml]. Irvine, CA: University of California, School of Information and Computer Science.

32. Buza, Krisztian. "Feedback prediction for blogs." Data analysis, machine learning and knowledge discovery. Springer, Cham, 2014. 145-152.

33. Nugteren, Cedric, and Valeriu Codreanu. "CLTune: A generic auto-tuner for OpenCL kernels." Embedded Multicore/Many-core Systems-on-Chip (MCSoC), 2015 IEEE 9th International Symposium on. IEEE, 2015.

34. Bertin-Mahieux, Thierry, et al. "The Million Song Dataset." Ismir. Vol. 2. No. 9. 2011.

35. Shannon, Paul, et al. "Cytoscape: a software environment for integrated models of biomolecular interaction networks." Genome research 13.11 (2003): 2498-2504.

36. Rosenbrock, HoHo. "An automatic method for finding the greatest or least value of a function." The Computer Journal 3.3 (1960): 175-184.

37. Dolan, Elizabeth D., and Jorge J. Mor. "Benchmarking optimization software with performance profiles." Mathematical programming 91.2 (2002): 201-213. 